\title{
Análisis de las competencias digitales de estudiantes de ingeniería de una universidad pública peruana
}

\author{
Analysis of digital skills of engineering students of a Peruvian public university
}

\author{
Segundo Agustín García Flores ${ }^{1}$ \\ https://orcid.org/0000-0001-8587-3211 \\ Universidad Alas Peruanas, Perú
}

Recibido: 01-08-2019

Aceptado: 16-12-2019

\section{Cita Recomendada}

García, S. (2019). Análisis de las competencias digitales de estudiantes de ingeniería de una universidad pública peruana. Hamut'ay, 6(3), 114-125. http://dx.doi.org/10.21503/hamu.v6i3.1852

\section{Resumen}

En este artículo partimos del supuesto que los estudiantes de ingeniería de una Universidad pública poseen competencias digitales para el manejo y uso eficaz de las TIC en la búsqueda y tratamiento de la información, la comunicación social y el aprendizaje. Por ello, se realizó una investigación descriptiva de corte cuantitativo basado en la percepción del estudiante. Se aplicó un cuestionario COBADI 2013 sobre competencias digitales básicas. La muestra fue de 140 estudiantes de las asignaturas de matemática del I, III y IV ciclo de estudios. Los resultados indican que el $70 \%$ de los estudiantes se perciben con una competencia digital en los niveles 2 y 3 . En la cuarta dimensión, el $55.8 \%$ de los estudiantes se perciben con una competencia digital alta $(37.9 \%$ nivel 3 y $17.9 \%$ nivel 4); mientras que la segunda dimensión, el $56.5 \%$ de los estudiantes tienen una percepción por debajo de la media en su competencia digital (8.6\% nivel 1 y 47.9\% nivel 2). Los estudiantes de 21 a 25 años con desempeños esperados que se manifiestan en un dominio completo al aplicar herramientas tecnológicas en su aprendizaje y en coherencia con las exigencias sociales. Se sugiere incorporar estrategias docentes para el desarrollo de las competencias tecnológicas en el aula.

Palabras Clave: competencia digital, formación profesional, fuente de información, tecnología.

\section{Abstract}

In this article, we start from the assumption that engineering students from a public University possess digital competences for the effective management and use of ICTs in the search and treatment of information, social communication and learning. Therefore, we carried out a descriptive research with a quantitative approach, based on the perception of the student. A 2013 COBADI questionnaire on basic digital skills was applied. The sample consisted of 140 students of the mathematics subjects of the I, III and IV cycle of studies. The results indicate that $70 \%$ of the students are perceived themselves with a digital competence in levels 2 and 3. In the fourth dimension, 55.8\% of

1 Maestro en Docencia Universitaria y Gestión Educativa. Licenciado en Matemáticas. Diplomado en Estadística Aplicada. Correo electrónico: sgflores13@gmail.com 
the students are perceived with a high digital competence (37.9\% level 3 and $17.9 \%$ level 4 ); while in the second dimension, $56.5 \%$ of the students have a perception below average in their digital competence (8.6\% level 1 and $47.9 \%$ level 2). Students aged 21 to 25 with expected performances who manifest themselves in a complete domain when applying technological tools in their learning and in coherence with social demands. It is suggested to incorporate teaching strategies for the development of technological skills in the classroom.

Keywords: digital competence, professional training, source of information, technology

\section{INTRODUCCIÓN}

En el siglo XXI, el uso creciente de dispositivos tecnológicos ha cambiado el panorama de la participación ciudadana (Ferrari, 2013), el trabajo, la interrelación social y la Educación (tanto a nivel de instituciones educativas como en las características y desempeños de los estudiantes). En ese contexto, la universidad tiene una gran responsabilidad social en la formación de calidad de los profesionales que la sociedad requiere, en la producción de conocimiento de alto nivel derivado de la investigación que contribuya en innovación y desarrollo. Por ello, la formación de futuros ingenieros debe tener una competencia clave: la digital, que no solo se refiere a poseer habilidades, asimilar contenidos y tener valores entorno a las TIC, como por ejemplo: Conocimientos para acceder y navegar en internet, búsqueda con distintos navegadores, uso de hipervínculos, elaboración y uso de formularios en línea, seguridad en la Web, etc., para la generación de nuevos productos y responder a desafíos tecnológicos de su área, sino a su aplicación en la resolución exitosa de una tarea (González-Zabala, Galvis- Lista, \& Sánchez-Torres, 2015).

Por otro lado, dado que se puede disponer de abundante información en milésimas de segundo, los estudiantes enfrentan el reto de transformarlos en conocimiento (desarrollo cognitivo, procedimental y actitudinal). $\mathrm{Al}$ respecto, aunque manejan fácilmente aplicativos para redacción de textos, elaborar presentaciones multimedia, hoja de cálculo, aún están en una fase incipiente en el manejo de recursos como las Wikis, Podcasts, mapas conceptuales y mentales, etc., relacionadas con la Web 2.0 (Castellanos, Sánchez \& Calderero, 2017), así como el no poseer un dominio para producir contenidos nuevos y difundirlos a través de redes de colaboración.

Como se evidencia en investigaciones de los últimos 15 años, sobre competencias digitales de jóvenes que afirman tener un nivel alto no están soportados empíricamente (Acosta-Silva, 2017). Al respecto, Chiecher \& Melgar (2018) establecen que los jóvenes universitarios no desarrollan adecuadamente la habilidad de escribir y editar en forma colaborativa usando tecnologías como Google drive. La implementación de innovaciones en este sentido permite, que un $85 \%$ lo valore positivamente para su uso en diversos contextos.

Desde la óptica de la docencia, a pesar de que el estudiante usa muchas tecnologías, tiene facilidad para acceder a información en Internet, es multitarea, pero tiene problemas en labores de lectura, escritura y reflexión crítica (Chiecher \& Lorenzati, 2017). Asimismo, utiliza e-books para tareas relacionadas con investigación formativa y prefieren leer materiales impresos en lugar de leerlos en una pantalla (Davidovitch, 2017).

De lo expresado, hay un interés en estudiar ¿Cuáles son las competencias digitales que emplean los estudiantes de ingeniería de una Universidad pública en el manejo y uso de las Tecnologías de la Información y las Comunicaciones (TIC) en la búsqueda y tratamiento de la información, la comunicación social y el aprendizaje?

Y como objetivo general se consideró el analizar las competencias digitales de los estudiantes de ingeniería de una Universidad pública utilizadas 
en la búsqueda, selección, análisis, interpretación, comunicación de la información y el aprendizaje. Siendo los objetivos específicos: i. Identificar el grado de conocimiento y formación sobre competencia digital que tienen los estudiantes de Ingeniería. ii. Reconocer la actividad más relevante que realizan los estudiantes de Ingeniería al buscar y tratar la información. iii. Identificar la dimensión más relevante de la Competencia digital de los estudiantes de Ingeniería. iv. Identificar el grupo etario de mejor desempeño en competencias digitales. Para lo cual se planteó como hipótesis general: Los estudiantes de Ingeniería con edades entre 21 y 25 años de edad son los que poseen una competencia digital de nivel alto en el manejo y uso eficaz de las TIC en la búsqueda y tratamiento de la información, la comunicación social y el aprendizaje.

\section{La Competencia Digital}

Definir la Competencia Digital es complejo, sin embargo, encontramos cuatro aspectos fundamentales intervinientes: lo cognitivo (conocimientos que el estudiante adquiere); la aplicación (práctica que el estudiante es capaz de realizar); la realización (valores y posturas) y el desempeño (básico, intermedio y avanzado) (Pech \& Prieto, 2016). Al respecto, en España, el Instituto Nacional de Tecnologías Educativas y Formación del Profesorado (INTEF) reporta que los conocimientos, destrezas y actitudes (valores) vinculados a las habilidades digitales se concentran en 5 áreas y 6 niveles competenciales por cada una de las 21 que conforman el Marco Común de Competencia Digital Docente (INTEF, 2017). Así, en particular, Valverde-Crespo, Pro-Bueno, \& González-Sánchez, (2018) describe las subcompetencias asociadas al área de información, las mismas que se circunscriben en la competencia digital y proyectan hacia áreas diversas para resolver problemas que involucran lo personal y social.

Anteriores estudios sistematizan el progresivo desarrollo de la competencia digital a partir de la idea de búsqueda de información, pasando por las dimensiones: tecnológicas, informacional, comunicativa, habilidad cognitiva y cultura digital, hasta la disposición de habilidades de búsqueda, obtención, procesamiento, comunicación y transformación en nuevo conocimiento utilizando habilidades como: dominio de lenguajes específicos, dominio y manejo de recursos y sistemas tecnológicos, habilidades para resolver problemas y destrezas de razonamiento para tratar información en conocimiento (Telefónica, 2019).

En términos generales, la competencia digital es el "uso creativo, crítico y seguro de las tecnologías de información y comunicación para alcanzar los objetivos relacionados con el trabajo, la empleabilidad, el aprendizaje, el tiempo libre, la inclusión y la participación en la sociedad" (INTEF, 2017, p.12); por lo que poseer una competencia digital asegura al que la posee el manejo diligente de tecnologías digitales en la ejecución de labores en el trabajo, el aprendizaje, el tiempo libre y la participación social (Avitia \& Uriarte, 2017; Ilomaki, Paavola, Lakkala \& Kantosalo, 2014).

\section{Competencia digital y aprendizaje}

El docente enfrenta el reto de dirigir a un estudiante cada vez más vinculado a Internet, familiarizado con la tecnología y que participa en la red con una identidad, colaborando con la construcción de esta cultura digital, por lo que se ha visto obligado a organizar actividades usando las tecnologías para mejorar su intervención en el aula. En consecuencia, asumir la enseñanza digital y el aprendizaje a lo largo del proceso educativo tiene su sustento en el conocimiento inicial de las habilidades digitales de los estudiantes, especialmente si son de ingeniería, debido a que estos están inmersos en este tipo de recursos, que migran y se actualizan constantemente.

En términos de tecnología educativa, los universitarios tienen destrezas y habilidades digitales heterogéneas, debido a que abordan su uso de distinta manera y suman experiencias diversas, según sus estilos de aprendizaje y actividades académicas. En un estudio realizado por Andrew, Taylorson, Langille, Grange \& Williams, (2018) evaluaron las actitudes de estudiantes universitarios en la integración efectiva de tecnologías para el desarrollo de tareas de aula, encontrando que los estudiantes al utilizar dichas tecnologías creen que estas apoyan su aprendizaje y los habilitan para su poste- 
rior ejercicio profesional.

Para Díaz (2016); Marín, Vidal, Peirats, \& San Martín (2019), la competencia digital es transversal, es decir, no se tiene que trabajar de manera independiente en una asignatura, sino, más bien, integradas a todas las asignaturas. Por un lado, si las tecnologías las empleamos solo para exponer nuestros conocimientos, esperamos que los estudiantes aprendan por recepción; sin embargo, ellos pueden aprender descubriendo, explorando mediante los recursos Web y los propios de su plataforma que la universidad dispone, lo que permite que el estudiante aprenda usando las tecnologías de modo dinámico (Díaz, 2016).

Para la adquisición de la competencia digital se requiere apoyarse en la tutoría y monitoreo desde la aplicación práctica y la ejecución planificada de actividades, considerando el aprendizaje grupal y la forma en que asimila el estudiante (Hernández-Sánchez, Quijano \& Pérez, 2019). Las tareas que se planifiquen deben tener distintos niveles de complejidad (su desarrollo debe considerar aspectos de la competencia digital), que signifique el uso de tecnologías digitales simples hasta la redacción de un ensayo como parte de un proyecto grupal (colaborativo) que tengan que defender. Tener en cuenta que el uso de las TIC es influido de manera significativa por factores de tipo motivacional de los estudiantes (Ramírez \& Barragán, 2018).

El aprendizaje del estudiante y las habilidades adquiridas están en relación con la empleabilidad en su carrera, con el desarrollo de hábitos que le permite seguir una trayectoria continua de aprendizaje, y la utilidad de las TIC en el desempeño de aula de los docentes y en el apoyo de tareas de retroalimentación en casos de estudiantes con bajo rendimiento.

\section{Futuros ingenieros y sus competencias digitales}

En el Perú, la formación del capital humano en tecnologías digitales de última generación se realiza en áreas como robótica, inteligencia artificial (IA), big data, a través de las 20 universidades mejor posicionadas (Katz, 2018). El aporte al crecimiento económico del país se basa en la per- tinencia de su oferta educativa, de su inmersión satisfactoria en el mercado laboral y los sectores productivos.

En esa línea de ideas, la universidad se preocupa en construir un entorno de formación equilibrado que genere procesos formativos orientados al desarrollo de un profesional con capacidades humanas plenas y que contribuya con su talento al desarrollo humano y económico de su entorno (Capote, Rizo, \& Bravo, 2016). El proceso de transformación digital que afecta la industria vuelve al mercado laboral más exigente. Es decir, para optimizar la empleabilidad, la formación debe centrarse en habilidades digitales (Álvarez-Flores, Núñez-Gómez, \& Crespo, 2017). Por ello, es preocupación creciente el impacto de la IA en el empleo; la automatización cada vez más extendida preocupa respecto al desplazamiento de los profesionales del mercado laboral (Telefónica, 2019).

En esa perspectiva, es vital la definición del perfil del ingeniero que deseamos formar. Es decir, cuáles son aquellas características de los egresados expresada en términos de las competencias que posee (entre ellas las digitales) para desempeñarse eficientemente en su profesión. La literatura reporta que algunas habilidades digitales serán más importantes que otras en la demanda laboral futura, entre ellas, las que resuelven problemas y ayudan a crear contenido digital, como el diseño de ingeniería, arquitectura, software, análisis de datos, etc. (Djumalieva \& Sleeman, 2018).

El proceso de diseño y gestión curricular para formar ingenieros de calidad, con base en matemáticas, física, química, computación, etc., debe procurar la creatividad y promover las habilidades de pensamiento crítico que permite afrontar y dar solución (basado en la ciencia y la tecnología) a problemas (cada vez más complejos) con autonomía y autodeterminación (Capote, Rizo, \& Bravo, 2016). Por lo que, formar un ingeniero requiere una base teórica sólida, el manejo y uso de técnicas e instrumentos, así como un conjunto de valores y actitudes que le permitan un trabajo eficaz en la sociedad marcada por las tecnologías y la información (Pérez, 2017; González, Nieto, Montenegro \& López, 2018). 
La Cuarta revolución industrial tiene implicancias a considerar en los diseños curriculares y planes de estudios de ingeniería, como, por ejemplo: "Competencias analíticas avanzadas (Big Data), Simulación avanzada y modelado virtual de plantas, competencias en ingeniería de computación, habilidades en la Interfaz Hombre - Máquina, (...)" (Carvajal, 2017, p.1). Si a ello le sumamos el Internet de las cosas (IoT), Ciberseguridad en protección de datos, computación en la nube, diseño e impresión 3D, realidad aumentada, nuevas competencias profesionales son requeridas, especialmente las digitales. En este punto, vale preguntarse, ¿Qué características debe tener la formación del ingeniero en el escenario digital? Según Carvajal (2017), se debe educar en: nuevos lenguajes de programación; Innovación empresarial como centro de la revolución tecnológica, y Learning Analytics. Asimismo, plantea una estructura de planes de estudios centrado en las competencias TIC concebir-diseñar-implementar-operar, las cuales considera transversales.

Desde la experiencia de los estudiantes de ingeniería se construye a partir de su interacción con las tecnologías, sus sentimientos, el contexto de aprendizaje y sus percepciones (Ramírez, \& Barragán, 2018). La práctica académica de los estudiantes recibe la influencia del uso innovador de las Tecnologías con las cuales se comunica y aprende (Zempoalteca, Barragán, González \& Guzmán, 2017).

\section{Materiales y Métodos}

\section{Participantes}

La población fue conformada por estudiantes de una Facultad de Ingeniería Pesquera y de Alimentos de una Universidad Pública de Perú, de I a IV ciclo de las carreras de Ingeniería Pesquera e Ingeniería de Alimentos, durante el semestre 2018-B. La edad de la población fue entre 17 a 31 años. Todos los estudiantes tienen una tarjeta de control médico, otorgado por la Oficina de Bienestar Universitario (OBU), y un certificado que es requisito para la matricula. La mayoría reside fuera de la Región Callao y viven con sus padres.
El tamaño de la muestra se determinó con $95 \%$ de confianza y una precisión de 5\%. La muestra fue de 140 estudiantes matriculados en las asignaturas: Matemática I (32.1\%), Matemática III (36.4\%) y Matemática IV (31.4\%) que forman parte de los planes curriculares de ambas carreras.

La edad media muestral es aproximadamente de 21 años. El $57.9 \%$ son hombres y el $42.1 \%$ son mujeres; el $52.1 \%$ tienen menos de 21 ańos, el $41.4 \%$ entre 21 y 25 , el $5.7 \%$ más de 26 años de edad; el $44.3 \%$ corresponden a Ingeniería Pesquera y el $55.7 \%$ estudian Ingeniería de Alimentos.

\section{Instrumento}

Mediante una encuesta, aplicada como auto empadronamiento, se recogen los datos. $\mathrm{Al}$ respecto, la encuesta es definida como "la técnica de obtención de datos mediante la interrogación a sujetos que aportan información relativa al área de la realidad a estudiar" (Yuni \& Urbano 2014, p.65).

Para responder al objetivo, seguimos a Moreno-Fernández, Moreno-Crespo \& Hunt-Gómez (2018), al aplicar el cuestionario "Competencias Básicas Digitales 2.0 de estudiantes universitarios" COBADI 2013. Este cuestionario utiliza una escala de 1 (mínimo) a 4 (máximo) para dar valor a cada uno de los ítems, se incluye NS/NC/ NA como opción para no sé, no conozco y no aplica. Los ítems que conforman cada una de las dimensiones del cuestionario se observan en la Tabla 1. La competencia digital de los estudiantes se distribuyó por niveles, siguiendo a Gallardo-Echenique, Poma, \& Esteve (2018). La determinación de los niveles de la competencia digital se realizó siguiendo los cuartiles para los datos de la variable:

Nivel 1: desempeño esperado en estudiantes de ingeniería que se inician en el uso de TIC.

Nivel 2: describe desempeños esperados de estudiantes de ingeniería que progresivamente van ganando experiencia y flexibilidad en el uso de TIC.

Nivel 3: describe desempeños esperados que evidencian que los estudiantes de ingeniería usan las TIC de modo eficiente en su aprendizaje. 
Nivel 4: describe desempeños esperados que se manifiesta en un dominio completo al aplicar herramientas tecnológicas en su aprendizaje y en coherencia con las exigencias sociales.

Tabla 1

Dimensiones del Cuestionario Competencias Básicas Digitales COBADI 2013

\begin{tabular}{|c|c|c|}
\hline & Dimensiones & Ítems \\
\hline I & $\begin{array}{l}\text { Competencias en Conocimiento y uso } \\
\text { de las TIC en la Comunicación Social y } \\
\text { aprendizaje colaborativo. }\end{array}$ & $\begin{array}{l}1,2,3,4,5,6 \\
7,8,9,10,11 \\
12,13\end{array}$ \\
\hline ॥ & $\begin{array}{l}\text { Competencias del uso de las TIC para la } \\
\text { búsqueda y tratamiento de la informa- } \\
\text { ción. }\end{array}$ & $\begin{array}{l}1,2,3,4,5,6 \\
7,8,9\end{array}$ \\
\hline III & $\begin{array}{l}\text { Competencias interpersonales en el uso } \\
\text { de las TIC en el contexto universitario. }\end{array}$ & $1,2,3,4$ \\
\hline IV & $\begin{array}{l}\text { Herramientas virtuales y de comunica- } \\
\text { ción social de la Universidad }\end{array}$ & $1,2,3,4$ \\
\hline
\end{tabular}

Fuente: Elaboración propia (2019).

Respecto a la validez y confiabilidad, es un instrumento desarrollado por el investigador Miguel Zapata Ross, patentado con registro No. 2970648.

\section{Tipo y diseño}

Esta investigación de tipo cuantitativa es de nivel descriptivo, transversal. Al respecto, estos estudios buscan "especificar las propiedades, las características y los perfiles de personas, grupos, comunidades, procesos, objetos o cualquier otro fenómeno que se someta a un análisis" (Hernández, Fernández \& Baptista, 2014, p.92).

\section{Procedimiento}

1. Definición del contexto del estudio y selección de los estudiantes que participaron de la investigación. Fueron consideradas las aulas que habían concluido con la actividad de investigación formativa en matemática.

2. Aplicación en forma física del cuestionario COBADI 2013. Se dieron las instrucciones y aclaración de dudas en cada aula antes de la ejecución del instrumento.

3. Los registros fueron exportados a un archivo
Excel, para organizarlos y proceder al procesamiento y análisis de datos mediante software estadístico.

4. Las conclusiones fueron sistematizadas y permitieron la redacción del artículo de investigación.

\section{Confidencialidad}

Los estudiantes participaron voluntariamente, después de haber sido informados de los objetivos de la investigación. La encuesta fue anónima, lo que no representaba ningún riesgo para la recolección de los datos que proporcionaran y que no existía beneficio económico alguno por los productos o resultados de la investigación.

\section{Resultados}

Los datos de la Tabla 2 muestran que 128 (91.4\%) estudiantes no han recibido formación sobre Web 2.0 o software social, mientras que $12(8.6 \%)$ recibió algún tipo de capacitación (destacándose entre ellos el 50\% que cursaron matemática IV).

Tabla 2

Formación sobre Web 2.0 o software social según asignatura

\begin{tabular}{lrrrrrr}
\hline & \multicolumn{5}{c}{ ¿Has recibido formación sobre Web 2.00} \\
& No & $\%$ & \multicolumn{1}{c}{ Si } & \multicolumn{1}{c}{$\%$} & TOTAL & $\%$ \\
\cline { 2 - 7 } & \multicolumn{1}{c}{ Notware social? } \\
\hline Matematica 1 & 44 & 33.4 & 1 & 8.3 & 45 & 32.1 \\
\hline Matematica 2 & 46 & 35.9 & 5 & 41.7 & 51 & 36.4 \\
\hline Matematica 3 & 38 & 29.7 & 6 & 50.0 & 44 & 31.4 \\
\hline TOTAL & 128 & 100.0 & 12 & 100 & 140 & 100.0 \\
\hline
\end{tabular}

Fuente: Elaboración propia (2019).

El $82.9 \%$ de los estudiantes tiene ordenador (de los cuales el $60.3 \%$ son hombres y el $39.7 \%$ son mujeres), mientras que el $17.1 \%$ no lo tiene. Asimismo, el 54.3\% no tiene Tablet frente al 45.7\% que sí la tiene. Asimismo, se destaca que el $82.1 \%$ dispone de Internet en casa frente a un $17.9 \%$ que no tiene conexión. De la Tabla 3, res- 
pecto al lugar donde habitualmente se conecta a Internet, el $52.1 \%$ lo hace en casa (de los cuales el $59.3 \%$ son hombres y el $42.4 \%$ son mujeres), seguido del $30 \%$ que lo hace desde cualquier sitio debido a que dispone de Internet en su celular (33.9\% de las mujeres versus $27.2 \%$ de los hombres), un $15 \%$ se conecta en la universidad (22\% de las mujeres versus el $9.9 \%$ de los hombres) y un porcentaje muy bajo en otro sitio.

Tabla 3

Lugar de conexión a Internet según género

\begin{tabular}{lcccccc}
\hline & \multicolumn{5}{c}{ ¿Dónde te conectas habitualmente a Internet? } \\
\cline { 2 - 7 } & Hombre & $\%$ & Mujer & $\%$ & TOTAL & $\%$ \\
\hline En casa & 48 & 59.3 & 25 & 42.4 & 73 & 32.1 \\
\hline En la universidad & 8 & 9.9 & 13 & 22.0 & 21 & 15.0 \\
\hline $\begin{array}{l}\text { En cualquier sitio } \\
\text { (móvil con Internet) }\end{array}$ & 22 & 27.2 & 20 & 33.9 & 42 & 30.0 \\
\hline $\begin{array}{l}\text { En casa de amis- } \\
\text { tades }\end{array}$ & 0 & 0.0 & 1 & 1.7 & 1 & 2 \\
\hline En un cibercafé & 2 & 2.5 & 0 & 0.0 & 2 & 1.4 \\
\hline Otro & 1 & 1.2 & 0 & 0.0 & 1 & 0.7 \\
\hline TOTAL & 81 & 100 & 59 & 100 & 140 & 100 \\
\hline
\end{tabular}

Fuente: Elaboración propia (2019).

Tabla 4

Tiempo dedicado a navegar por Internet según género

\begin{tabular}{lcccccc}
\hline & \multicolumn{6}{c}{ ¿Cuánto tiempo dedicas a navegar por Internet? } \\
\cline { 2 - 7 } & Hombre & $\%$ & Mujer & $\%$ & TOTAL & $\%$ \\
\hline 1-3 horas/semana & 26 & 32.1 & 17 & 28.8 & 43 & 30.7 \\
\hline 4-9 horas/semana & 25 & 30.9 & 23 & 39.0 & 48 & 34.3 \\
\hline$>9$ horas/semana & 27 & 33.3 & 18 & 30.5 & 45 & 32.1 \\
\hline Otro & 3 & 3.7 & 1 & 1.7 & 4 & 2.9 \\
\hline TOTAL & 81 & 100 & 59 & 100 & 140 & 100 \\
\hline
\end{tabular}

Fuente: Elaboración propia (2019).

De Tabla 4, el tiempo semanal que dedican los estudiantes a navegar por Internet, el 30.7\% dedica entre 1 y 3 horas (de los cuales el $32.1 \%$ son hombres y el $28.8 \%$ son mujeres), el $34.3 \%$ entre 4 y 9 horas (de los cuales el $30.9 \%$ son hombres y el $30.5 \%$ son mujeres), el $32.1 \%$ más de 9 horas (de los cuales el $33.3 \%$ son hombres y el $42.4 \%$ son mujeres) y un porcentaje pequeño otro tiempo.

En la Tabla 5 se observa que el $66.7 \%$ de los estu- diantes que navegan en Internet entre 4 y 9 horas semanales lo hacen desde su casa. El 57.8\% de los que navegan más de 9 horas semanales lo hacen desde su celular pues disponen de Internet móvil versus el $11.1 \%$ que se conecta en la Universidad.

\section{Tabla 5}

Lugar de conexión habitual a Internet según el tiempo dedicado a navegar

\begin{tabular}{lcccccccccc}
\hline & \multicolumn{1}{c}{ Tiempo dedicado a navegar por Internet (horas/ } \\
& \multicolumn{10}{c}{ semana) } \\
& $1-3$ & $\%$ & $4-9$ & $\%$ & $>9$ & $\%$ & Otro & $\%$ & Total & $\%$ \\
\hline En casa & 25 & 58.1 & 32 & 66.7 & 14 & 31.1 & 2 & 50.0 & 73 & 52.1 \\
\hline $\begin{array}{l}\text { En la univer- } \\
\text { sidad }\end{array}$ & 11 & 25.6 & 5 & 10.4 & 5 & 11.1 & 0 & 0.0 & 21 & 15.0 \\
\hline $\begin{array}{l}\text { En cualquier } \\
\text { sitio (móvil } \\
\text { con Internet) }\end{array}$ & 6 & 14.0 & 9 & 18.8 & 26 & 57.8 & 1 & 25.0 & 42 & 30.0 \\
\hline Otro & 1 & 2.3 & 2 & 4.2 & 0 & 0.0 & 1 & 25.0 & 4 & 2.8 \\
\hline TOTAL & 43 & 100.0 & 48 & 100.0 & 45 & 100.0 & 4 & 100.0 & 140 & 100.0 \\
\hline
\end{tabular}

Fuente: Elaboración propia (2019).

En la Tabla 6, se destaca que el $72.9 \%$ de los estudiantes registran un tiempo de conexión a Internet superior a 5 horas semanales, que las dedican a buscar información para realizar las tareas universitarias, seguido de un $60 \%$ dedica menos de 5 horas semanales dedicadas a publicar fotografías o videos, el $57.1 \%$ se conecta más de 5 horas dedicadas a escuchar música. Finalmente, el 53.6\% no utiliza nunca su tiempo de conexión a Internet en jugar online.

Se aplica el análisis factorial sobre variables de tipo ordinal, de acuerdo con Morales (2013). La idoneidad de los datos para realizar este análisis se mide mediante la prueba de Kaiser-Meyer-Olkin (KMO), cuyo valor es 0.805 considerado notable. La prueba de esfericidad de Bartlett nos reporta que el modelo es adecuado con $\alpha=0.000$ $<0.05$ (Tabla 7).

En la determinación de la matriz de factores rotados se empleó los componentes principales y la rotación fue mediante varimax con normalización Kaiser, siguiendo a Henson \& Roberts (2006). Los Coeficientes fueron ordenados por tamaño superiores a 0.40 . La matriz de rotación otorga hasta 9 factores, sin embargo, de acuerdo a Costello \& Osborne (2003) solo tomamos los factores 
del 1 al 4 debido a que los otros factores tienen menos de tres ítems. Estos factores considerados constituyen el $44.27 \%$ de la varianza explicada (Tabla 8). Así, el primer factor sólido está definido por más de 5 ítems con pesos mayores a 0.50 .

\section{Tabla 6}

Tiempo de conexión a Internet por cada actividad realizada por el estudiante (\%)

\begin{tabular}{|c|c|c|c|}
\hline & Nada & Poco & Mucho \\
\hline Ver programas de televisión & 40.0 & 55.7 & 4.3 \\
\hline Escuchar música & 5.0 & 37.9 & 57.1 \\
\hline $\begin{array}{l}\text { Informarme sobre temas que me interesan a } \\
\text { nivel académico y profesional }\end{array}$ & 4.3 & 45.7 & 50.0 \\
\hline Jugar online & 53.6 & 32.1 & 14.3 \\
\hline $\begin{array}{l}\text { Buscar información para realizar las tareas } \\
\text { universitarias }\end{array}$ & 2.1 & 25.0 & 72.9 \\
\hline Publicar fotografías/videos & 26.4 & 60.0 & 13.6 \\
\hline Bajar música, películas, juegos, etc. & 21.4 & 53.6 & 25.0 \\
\hline $\begin{array}{l}\text { Hablar con los amigos a través de redes so- } \\
\text { ciales, chat... }\end{array}$ & 3.6 & 44.3 & 52.1 \\
\hline $\begin{array}{l}\text { Buscar amigos/as nuevos mediante el uso de } \\
\text { redes sociales (Facebook, Tuenti, Twitter,...) }\end{array}$ & 39.3 & 43.6 & 17.1 \\
\hline $\begin{array}{l}\text { Trabajar en grupo con compañeros/as de cla- } \\
\text { se para realizar tareas académicas }\end{array}$ & 6.4 & 46.4 & 47.1 \\
\hline
\end{tabular}

Fuente: Elaboración propia (2019).

Tabla 7

Prueba de KMO y Bartlett para el análisis factorial

\begin{tabular}{llr}
\hline $\begin{array}{l}\text { Medida Kaiser-Meyer-Olkin de adecuación de } \\
\text { muestreo }\end{array}$ & 0.805 \\
\hline $\begin{array}{l}\text { Prueba de esfericidad de } \\
\text { Bartlett }\end{array}$ & Aprox. Chi-cuadrado & 1697.570 \\
\cline { 2 - 3 } & Gl & 496 \\
\cline { 2 - 3 } & Sig. & 0.000 \\
\hline
\end{tabular}

Fuente: Elaboración propia (2019).

Tabla 8

Factores y porcentaje de varianza explicada

\begin{tabular}{llr}
\hline & Factores & $\begin{array}{c}\text { Varianza } \\
\text { explicada } \\
(\%)\end{array}$ \\
\hline F1 & Participación, comunicación y aprendizaje. & 23.977 \\
\hline F2 & Herramientas de comunicación. & 7.872 \\
\hline F3 & Resolución de problemas. & 7.105 \\
\hline F4 & Uso de buscadores y mensajería. & 5.314 \\
\hline & TOTAL & 44.268 \\
\hline
\end{tabular}

Fuente: Elaboración propia (2019).
La estimación de las habilidades digitales se realizó siguiendo a Organista, Sandoval, \& McAnally (2016). Los estudiantes se perciben con una competencia digital media de 2.66 sobre 4 . En la Tabla 9, destaca la segunda dimensión con una media de 2.86 frente a la cuarta dimensión Herramientas virtuales y de comunicación social de la Universidad con una media de 2.49 , la más baja (Tabla 9).

\section{Tabla 9}

Percepción de estudiantes de ingeniería sobre su competencia digital

\begin{tabular}{lcccl}
\hline & N & Suma & Media & DS \\
\hline $\begin{array}{l}\text { B1: Competencias en Co- } \\
\text { nocimiento y uso de las }\end{array}$ & 140 & 385.62 & 2.7544 & 1.07026 \\
$\begin{array}{l}\text { TIC en la Comunicación } \\
\text { Social y aprendizaje cola- } \\
\text { borativo. }\end{array}$ & & & & \\
\hline $\begin{array}{l}\text { B2: Competencias del } \\
\text { uso de las TIC para la }\end{array}$ & 140 & 401.36 & 2.8669 & 1.32794 \\
búsqueda y tratamiento de & & & & \\
la información. & & & & \\
\hline $\begin{array}{l}\text { B3: Competencias } \\
\text { interpersonales en el uso } \\
\text { de las TIC en el contexto }\end{array}$ & 140 & 357.50 & 2.5536 & .67775 \\
universitario & & & & \\
\hline $\begin{array}{l}\text { B4: Herramientas virtuales } \\
\text { y de comunicación social }\end{array}$ & 140 & 349.50 & 2.4964 & .87839 \\
de la Universidad & & & & \\
\hline $\begin{array}{l}\text { Competencia digital total } \\
\text { demp }\end{array}$ & 140 & 373.49 & 2.6678 & .67378 \\
\hline
\end{tabular}

Fuente: Elaboración propia (2019).

De la Tabla 10, el 70\% de los estudiantes se perciben con una competencia digital en los niveles 2 y 3. Se destaca los resultados de la cuarta dimensión, el $55.8 \%$ de los estudiantes se perciben con una competencia digital alta (37.9\% nivel 3 y $17.9 \%$ nivel 4); mientras que la segunda dimensión, el $56.5 \%$ de los estudiantes tienen una percepción por debajo de la media en su competencia digital (8.6\% nivel 1 y $47.9 \%$ nivel 2 ).

De la Tabla 11, en cuanto a la edad de los estudiantes y su relación con la competencia digital, el $57.1 \%$ de los estudiantes con competencia digital de nivel 1 tiene su edad inferior a 21 ańos. En dicho nivel, el 4.8\% supera los 26 ańos. El 67.3\% de los estudiantes con competencia digital de nivel 2 tiene una edad menor a 21 años. En este nivel, el 2.0\% supera los 26 años. En el nivel 3, el 
$51.0 \%$ es menor a 21 ańos; mientras que el $8.2 \%$ entre ellos tienen más de 26 años. El 71.4\% de los estudiantes con competencia digital de nivel 4 tiene una edad entre 21 a 25 años. Este porcentaje es menor $14.0 \%$ en los otros grupos etarios. Las pruebas chi-cuadrado para las variables de la Tabla 11 con el valor del estadístico del contraste 17.868 (bilateral). El p-valor es 0.007. A un nivel de significación de 0.05 , la edad del estudiante se relaciona con la competencia digital total.

\section{Tabla 10}

Distribución de la competencia digital de los estudiantes de ingeniería en porcentaje

\begin{tabular}{lcccc}
\hline & Nivel 1 & Nivel 2 & Nivel 3 & Nivel 4 \\
\hline B1: Competencias en Co- & 12.1 & 41.4 & 36.4 & 10.0 \\
nocimiento y uso de las & & & & \\
TIC en la Comunicación & & & & \\
Social y aprendizaje cola- & & & & \\
borativo.
\end{tabular}

\begin{tabular}{lcccc}
\hline $\begin{array}{l}\text { B2: Competencias del } \\
\text { uso de las TIC para la } \\
\text { búsqueda y tratamiento }\end{array}$ & 8.6 & 47.9 & 37.9 & 5.7 \\
de la información. & & & & \\
\hline $\begin{array}{l}\text { B3: Competencias inter- } \\
\text { personales en el uso de }\end{array}$ & 13.6 & 38.6 & 29.3 & 18.6 \\
las TICS en el contexto \\
universitario
\end{tabular}

Fuente: Elaboración propia (2019).

Respecto a la relación entre asignatura de matemática y la competencia digital, se tiene que el 47.6\% de los estudiantes con competencia digital de nivel 1 cursaron matemática 1 . Este porcentaje es menor, $19.0 \%$ entre los que cursaron matemática 4 . El 40.8\% de los estudiantes con competencia digital de nivel 2 cursaron matemática 1. Este porcentaje es menor, $20.4 \%$ entre los que cursaron matemática 4 . El $42.9 \%$ de los estudiantes con competencia digital de nivel 3 cursaron matemática 3. Este porcentaje es menor, $28.6 \%$ en las otras asignaturas. El $76.2 \%$ de los estudiantes con competencia digital de nivel 4 cursaron matemática 4 . Este porcentaje es menor, $4.8 \%$ en los que cursaron matemática 1 (Tabla 12). Las pruebas chi-cuadrado para las variables de la Tabla 12 con el valor del estadístico del contraste 26.664 (bila- teral). El p-valor es 0.000 . Por lo tanto, a un nivel de significación de 0.05 , la asignatura cursada por el estudiante se relaciona con la competencia digital total.

\section{Tabla 11}

Edad del estudiante por niveles de Competencia Digital Total

\begin{tabular}{lcccccccccc}
\hline & \multicolumn{10}{c}{ Competencia Digital Total } \\
& Ni- & $\%$ & Ni- & $\%$ & Ni- & $\%$ & Ni- & $\%$ & Total & $\%$ \\
& vel & & vel & & vel & & vel & & & \\
& 1 & & 2 & & 3 & & 4 & & & \\
\hline$<21$ & 12 & 57.1 & 33 & 67.3 & 25 & 51.0 & 3 & 14.3 & 73 & 52.1 \\
\hline 21 a 25 & 8 & 38.1 & 15 & 30.6 & 20 & 40.8 & 0 & 0.0. & 21 & 15.0 \\
\hline$>26$ & 6 & 14.0 & 9 & 18.8 & 26 & 57.8 & 1 & 25.0 & 42 & 30.0 \\
\hline Total & 21 & 100.0 & 49 & 100.0 & 49 & 100.0 & 21 & 100.0 & 140 & 100.0 \\
\hline
\end{tabular}

Fuente: Elaboración propia (2019).

\section{Tabla 12}

Asignatura de matemática por niveles de Competencia Digital Total

\begin{tabular}{|c|c|c|c|c|c|c|c|c|c|c|}
\hline & \multicolumn{10}{|c|}{ Competencia Digital Total } \\
\hline & $\begin{array}{c}\mathrm{Ni}- \\
\text { vel } \\
1\end{array}$ & $\%$ & $\begin{array}{c}\mathrm{Ni}- \\
\text { vel } \\
2\end{array}$ & $\%$ & $\begin{array}{c}\mathrm{Ni}- \\
\text { vel } \\
3\end{array}$ & $\%$ & $\begin{array}{c}\mathrm{Ni}- \\
\text { vel } \\
4\end{array}$ & $\%$ & $\begin{array}{l}\text { To- } \\
\text { tal }\end{array}$ & $\%$ \\
\hline Matemática 1 & 10 & 47.6 & 20 & 40.8 & 14 & 28.6 & 1 & 4.8 & 45 & 32.1 \\
\hline Matemática 2 & 7 & 33.3 & 19 & 38.8 & 21 & 42.9 & 4 & 19.0 & 51 & 36.4 \\
\hline Matemática 3 & 4 & 19.0 & 10 & 20.4 & 14 & 28.6 & 16 & 76.2 & 44 & 31.4 \\
\hline Total & 21 & 100.0 & 49 & 100.0 & 49 & 100.0 & 21 & 100.0 & 140 & 100.0 \\
\hline
\end{tabular}

Fuente: elaboración propia (2019)

\section{Discusión y Conclusiones}

Del análisis de resultados se evidencia que la mayoría de estudiantes de ingeniería no han recibido formación sobre las herramientas de la Web 2.0 o software social; tienen computadora con conexión a Internet en casa desde donde habitualmente se conectan, lo cual es similar a lo encontrado por Castellanos, Sánchez \& Calderero (2017) quienes obtuvieron que la mayoría (99.7\%) de estudiantes tiene en casa ordenador conectado a Internet, contrastando con González, et al. (2018) quienes hallaron que el $21.6 \%$ de los estudiantes consultados se conectaron a Internet diariamente desde su hogar. 
Respecto al tiempo utilizado a navegar por Internet, la mayoría se conecta más de 5 horas semanales, que las dedican a buscar información relevante para realizar las tareas universitarias; en este caso, relacionadas con la temática y el desarrollo del trabajo de investigación formativa de las asignaturas de matemáticas. Esto es distante del $56.3 \%$ que no participa o participa poco en actividades ejecutadas por la Universidad a través de redes sociales, encontrado por Cordero \& Mory (2018).

Evidenciamos que los estudiantes de ingeniería, mayoritariamente se perciben con una competencia digital en los niveles 2 y 3 . En cuanto a las dimensiones, destacamos que en la cuarta dimensión, la mayoría se percibe con una competencia digital alta $(37.9 \%$ nivel 3 y $17.9 \%$ nivel 4); mientras que, en la segunda dimensión la mayoría de estudiantes se perciben con una competencia digital media-baja (8.6\% nivel 1 y $47.9 \%$ nivel 2), contrastando con los resultados de Gallardo-Echenique et al. (2018) quienes encontraron que el $58 \%$ de los estudiantes se perciben con una competencia digital alta.

Respecto a la hipótesis planteada, la prueba chi-cuadrado para las variables de la Tabla $11 \mathrm{da}$ un valor del estadístico del contraste 17.868 (bilateral) y un p-valor de 0.007 . Por lo tanto, a un nivel de significación de 0.05 , se concluye que la edad del estudiante se relaciona con la competencia digital total. Se evidencia que los estudiantes con edades entre 21 y 25 años de edad son los que poseen una competencia digital de nivel alto en el manejo y uso eficaz de las TIC en la búsqueda y tratamiento de la información, la comunicación social y el aprendizaje.

Mientras que los estudiantes menores de 21 años se perciben en el primer nivel, lo que confirma los resultados de Castellanos et al. (2017). Es decir, a pesar que son los más activos en el uso de tecnologías no queda claro que las orienten hacia tareas académicas o a generación de contenidos ya que es un tema complejo, más bien son próximos a actividades sociales y lúdicas más que académicas vinculadas a sus procesos de enseñanza y aprendizaje.

Los resultados evidencian de manera significati- va que los estudiantes de matemática IV son los que desarrollan competencias digítales del nivel más alto. Vale decir, utilizan las TIC no solo para comunicarse de manera eficiente sino para la búsqueda y tratamiento de la información, propiciando aprendizajes grupales que son comunicados a través de exposiciones de su trabajo académico, coincidiendo con Islas \& Delgadillo (2016). Es posible que estos estudiantes tengan más cercanía con la tecnología valorándolo positivamente, según Chiecher \& Melgar (2018) y su permanencia superior a un año en la universidad, le de alguna experiencia en las asignaturas de matemáticas, lo cual confirma los resultados de Castellanos et al. (2017), Andrew, et al. (2018), Avitia \& Uriarte (2017) y Ilomaki, et al. (2014).

Esta investigación, evidencia que hay necesidades formativas (Moreno-Fernández, et al. 2018) en la integración de tecnologías con eficientes estrategias didácticas e intervención docente que facilite el desarrollo de habilidades digitales, su evaluación y mejore la propuesta formativa de la institución.

Este trabajo, aporta una primera exploración de cómo los estudiantes de ingeniería se perciben en relación al manejo y uso eficaz de las tecnologías digitales, sus comunicaciones en redes sociales y su aprendizaje. Asimismo, es necesario contrastar con mediciones del desempeño del estudiante de ingeniería en relación con actividades que aseguren su formación profesional para un mundo digital.

Se sugiere replicar el estudio en las otras ingenierías de la universidad dado que las distintas facultades presentan cursos comunes en los primeros cuatro semestres de formación. En perspectiva, se tiene la elaboración de un cuestionario sobre competencias digitales para estudiantes de ingeniería que tome en cuenta sus niveles de conocimiento sobre tecnologías con fines de certificación.

Se recomienda planificar una intervención docente que recoja y potencie los beneficios de integrar las TIC en el aprendizaje para promover y desarrollar habilidades digitales en estudiantes de ingeniería. 


\section{REFERENCIAS BIBLIOGRÁFICAS}

Acosta-Silva, D. A. (2017). Tras las competencias de los nativos digitales: avances de una metasíntesis. Revista Latinoamericana de Ciencias Sociales, Niñez y Juventud, 15(1), 71-489.

Álvarez-Flores, E. P., Núñez-Gómez, P., \& Crespo, C. R. (2017). Adquisición y caren-cia académica de competencias tecnológicas ante una economía digital. Revista Latina de Comunicación social, (72), 540-559. https://doi. org/10.4185/RLCS-2017-1178

Andrew, M., Taylorson, J., Langille, D. J., Grange, A., \& Williams, N. (2018). Student attitudes towards technology and their preferences for learning tools/devices at two universities in the UAE. Journal of Information Technology Education: Research, 17, 309-344. https://doi. org/10.28945/4111

Avitia, P., \& Uriarte, I. (2017). Evaluación de la habilidad digital de los estudiantes universitarios: estado de ingreso y potencial educativo. Pixel-Bit, 61, 1-13.

Capote, G. E., Rizo, N., \& Bravo, G. (2016). La formación de ingenieros en la actuali-dad. Una explicación necesaria. Revista Universidad y Sociedad, 8 (1), 21-28.

Carvajal, R., H., J. (2017). La Cuarta Revolución Industrial o Industria 4.0 y su Impac-to en la Educación Superior en Ingeniería en Latinoamérica y el Caribe. Universidad Antonio Nariño, Colombia.

Castellanos, A., Sánchez, C., \& Calderero, J. (2017). Nuevos modelos tecnopedagógi-cos. Competencia digital de los alumnos universitarios. Revista electrónica de investiga-ción educativa, 19 (1), 1-9.

https://doi.org/10.24320/redie.2017.19.1.1148

Chiecher, A. C., \& Melgar, M. F. (2018). ¿Lo saben todo? Innovaciones educativas orientadas a promover competencias digitales en universitarios. Apertura, 10 (2), 110-123. https://doi.org/10.32870/Ap.v10n2.1374

Chiecher, A., \& Lorenzati, K. (2017). Estudiantes y tecnologías. Una visión desde la "lente" de docentes universitarios. Revista Iberoamericana de Educación a Distancia, 20, (1), 261-282. https://doi.org/10.5944/ried.20.1.16334

Cordero, D., \& Mory, A. M. (2018). Pertenencia en la Competencia Digital en los estu-diantes de la Universidad Católica de Cuenca, un estudio descriptivo. Revista Científi-ca y Tecnológica UPSE, 5(1), 29-35.

https://doi.org/10.26423/rctu.v5i1.300

Costello, A. B., \& Osborne, J. W. (2003). Exploring best practices in Factor Analysis: Four mistakes applied researchers make. Paper presented at the Annual Meeting of the American Educational Research Association, April 21, 2003, Chicago, IL.

Davidovitch, N. (2017). The effect of technology on students' reading habits: Reading from a screen versus reading from paper. Pedagogical Sciences, 6, 91-97.

Díaz, R. T. (2016). Las competencias transversales, su per- tinencia en la integralidad de la formación de profesionales. Didasc@ lia: Didáctica y Educación, 7(6), 199-228.

Djumalieva, J., \& Sleeman, C. (2018). Which digital skills do you really need? London. Recuperado de https://www. nesta.org.uk/report/which-digital-skills-do-you-really-need.

España. Instituto Nacional de Tecnologías Educativas y Formación del Profesorado (INTEF) (2017). Marco Común de la Competencia Digital Docente. Octubre. 2017.

Ferrari, A. (2013). DIGCOMP: A framework for developing and understanding digital competence in Europe.

Gallardo-Echenique, E. E., Poma, A., \& Esteve, F. M. (2018). La competencia digital: análisis de una experiencia en el contexto universitario.

González, C. C., Nieto, Y. V., Montenegro, C. E., \& López, J. F. (2018). Sociedad de la tecnología la información y el conocimiento: tecnologías en la formación de ingenie-ros. Revista Ibérica de Sistemas e Tecnologías de Informação, (E15), 304-317.

González-Zabala, M. P., Galvis-Lista, E. A., \& Sánchez-Torres, J. M. (2015). Identifi-cación de factores que afectan el desarrollo de la inclusión digital. Revista Virtual Uni-versidad Católica del Norte, 1(44), 175-191.

Henson, R. K., \& Roberts, J. K. (2006). Use of Exploratory Factor Analysis in Pub-lished Research: Common Errors and Some Comment on Improved Practice. Educa-tional and Psychological Measurement, 66, 393-416 https://doi.org/10.1177/0013164405282485

Hernández, R., Fernández, C., \& Baptista, P. (2014). Metodología de la investigación, México DF, México: McGraw Hill.

Hernández-Sánchez, A., Quijano, R. \& Pérez, M. (2019). La formación digital del estu-diante universitario digital: competencias, necesidades y pautas de actuación. Hamut'ay, 6(1), 19-32. https://doi.org/10.21503/hamu.v6i1.1572

Ilomaki, L., Paavola, S., Lakkala, M., \& Kantosalo, A. (2014). Digital competence - an emergent boundary concept for policy and educational research. Education Information Technology, 1-25. https://doi.org/10.1007/s10639014-9346-4

Islas, C., \& Delgadillo, O. (2016). La inclusión de TIC por estudiantes universitarios: una mirada desde el Conectivismo. Apertura, 8(2), 116-129.

https://doi.org/10.32870/Ap.v8n2.845

Katz, R. L. (2018). Capital humano para la transformación digital en América Latina.

Marín, D., Vidal, M., Peirats, J., \& San Martín, Á. (2019). Competencia digital trans-versal en la formación del profesorado, análisis de una experiencia. Innoeduca. Interna-tional Journal of Technology and Educational Innovation, 5(1), 4-12.

https://doi.org/10.24310/innoeduca.2019.v5i1.4890

Morales, P. (2013). El análisis factorial en la construcción e interpretación de tests, esca-las y cuestionarios. Madrid: Universidad Pontificia Comillas. 
Moreno-Fernández, O., Moreno-Crespo, P., \& Hunt-Gómez, C., I. (2018). University students in southwestern Spain digital competences. Les Ulis: EDP Sciences. https:// doi.org/10.1051/shsconf/20184801012

Organista, J., Sandoval, M., \& McAnally, L. (2016). Estimación de las habilidades digi-tales con propósito educativo de estudiantes de dos universidades públicas mexicanas. Revista electrónica de tecnología educativa (57), 46-62. https://doi.org/10.21556/edutec.2016.57.673

Pech, S. J., \& Prieto, M. E. (2016). La medición de la competencia digital e informacio-nal. T. De León, J. García, \& E. Orozco, Desarrollo de las competencias para el Siglo XXI, 26-50.

Pérez, C. D. (2017). Enseñanza de las competencias de investigación: un reto en la ges-tión educativa. Atenas, 1(37), 1-14.

Ramírez, U. N., \& Barragán, J. F. (2018). Autopercepción de estudiantes universitarios sobre el uso de tecnologías digitales para el aprendizaje. Apertura (Guadalajara, Jal.), 10(2), 94-109. https://doi.org/10.32870/Ap.v10n2.1401

Sánchez-Robayo, B., \& Torres-Duarte, J. (2016). Aprender a investigar investigando. Realización de una propuesta de formación - Learn to investigate by investigating. Re-alization of a training proposal. Revista científica, 1(28), 17-31. https://doi.org/10.14483/udistrital.jour.RC.2017.28.a2

Telefónica, F. (2019). Sociedad digital en Espańa 2018. Fundación Telefónica.

Valverde-Crespo, D., Pro-Bueno, A. J., \& González-Sánchez, J. (2018). La competencia informacional-digital en la enseñanza y aprendizaje de las ciencias en la educación se-cundaria obligatoria actual: una revisión teórica. Revista Eureka sobre Enseñanza y Di-vulgación de las Ciencias, 2105-2105. https://doi.org/10.25267/Rev_Eureka_ensen_ divulg_cienc.2018.v15.i2.2105

Yuni, A., \& Urbano, A. (2014). Técnicas para investigar 2. Argentina: Brujas.

Zempoalteca, B; Barragán, J; González, J., \& Guzmán, T. (2017). Formación en TIC y competencia digital en la docencia en instituciones públicas de educación superior. Apertura, 9 (1), 80-96. https://doi.org/10.32870/Ap.v9n1.922 